\title{
Stress Hyperglycemia Ratio may Portend Poor Outcomes in COVID-19
}

\author{
Rohan Magoon $^{1} \odot$, Jes Jose ${ }^{2} \odot$
}

Keywords: Admission blood glucose, COVID-19, Diabetes mellitus, Poor outcomes, Relative hyperglycemia, Stress hyperglycemia ratio. Indian Journal of Critical Care Medicine (2021): 10.5005/jp-journals-10071-24007

Dear Editor,

Amidst an ardent interest in the pathophysiological and prognostic links between diabetes mellitus and coronavirus disease-2019 (COVID-19), the concept of stress hyperglycemia merits concurrent research attention. This becomes particularly relevant in the background of reports of an augmented disease severity in COVID-19 patients demonstrating elevated admission blood glucose levels, irrespective of the preexisting diabetic status. ${ }^{1,2}$

The absolute degree of admission hyperglycemia has been described to be associated with poor COVID-19 outcomes by Lazarus et al. (33\% increase in the risk of severe disease for every $1 \mathrm{mmol} / \mathrm{L}$ rise in the admission fasting blood glucose) in their doseresponse meta-analysis. ${ }^{1}$ Moreover, few independent researchers outline a worsened attributable prognosis (owing to admission hyperglycemia) in nondiabetics ahead of the diabetics. ${ }^{2}$

While hyperglycemia at presentation emerges as a pertinent prognostic finding, the findings of a French nationwide multicenter CORONADO study [severe acute respiratory syndrome coronavirus 2 (SARS-CoV-2) and diabetes outcomes] simultaneously undermine the isolated prognostic implications of the background glycemic control [assessed by glycated hemoglobin ( $\mathrm{HbA1c})] .{ }^{3}$ This highlights the outcome predictive significance of acute hyperglycemia over and above the chronic glycemic control, particularly in the absence of long-term adaptations to deal with the hyperglycemiaexacerbated constellation of endotheliitis, oxidative stress, and thrombosis (well-known features of a severe COVID-19 infection, inflammation being the common denominator). ${ }^{4}$

In this context, stress hyperglycemia ratio (SHR), introduced by Roberts et al., is computed as the ratio of admission blood glucose and the estimated average blood glucose derived from $\mathrm{HbA1c}$, thereby adjusting for the background glycemia adequately. Withstanding this fact, SHR can potentially be a superior marker of the relative extent of the stress glycemic response to the intercurrent critical illnesses, such as an underlying COVID-19 infection. ${ }^{5}$ The findings of Fabbri et al., revealing a significantly accentuated mortality prediction with an odds ratio of 5.25 and area under the receiver-operating characteristic curve of 0.864 attributable to a SHR $\geq 1.14$ in critically ill septic diabetics, bear testimony to the same. ${ }^{5}$ In addition, independent research groups also emphasize the importance of disease-related glycemic alterations in contemplating risk stratification of the critically ill based on their depiction of an improved inhospital mortality prediction with the inclusion of the component of stress-induced hyperglycemia to the Acute Physiology and Chronic Health Evaluation (APACHE II) score. ${ }^{5}$

Therefore, premised on the aforementioned discussion, future research endeavors focusing on the concept of relative
${ }^{1}$ Department of Cardiac Anaesthesia, Atal Bihari Vajpayee Institute of Medical Sciences, Dr Ram Manohar Lohia Hospital, New Delhi, India

${ }^{2}$ Department of Cardiac Anesthesiology, Sri Jayadeva Institute of Cardiovascular Sciences and Research, Bengaluru, Karnataka, India

Corresponding Author: Jes Jose, Department of Cardiac Anesthesiology, Sri Jayadeva Institute of Cardiovascular Sciences and Research, Bengaluru, Karnataka, India, Phone: +91 9741447662, e-mail: drjesjoseworkmail@gmail.com

How to cite this article: Magoon R, Jose J. Stress Hyperglycemia Ratio may Portend Poor Outcomes in COVID-19. Indian J Crit Care Med 2021;25(11):1329.

Source of support: Nil

Conflict of interest: None

hyperglycemia are advocated wherein an elevated SHR may portend poor outcomes in the setting of COVID-19.

\section{OrCID}

Rohan Magoon @ https://orcid.org/0000-0003-4633-8851

Jes Jose 으 https://orcid.org/0000-0003-1734-9519

\section{References}

1. Lazarus G, Audrey J, Wangsaputra VK, Tamara A, Tahapary DL. High admission blood glucose independently predicts poor prognosis in COVID-19 patients: a systematic review and dose-response metaanalysis. Diabetes Res Clin Pract 2021;171:108561. DOI: 10.1016/ j.diabres.2020.108561.

2. Cai Y, Shi S, Yang F, Yi B, Chen X, Li J, et al. Fasting blood glucose level is a predictor of mortality in patients with COVID-19 independent of diabetes history. Diabetes Res Clin Pract 2020;169:108437. DOI: 10.1016/j.diabres.2020.108437.

3. Cariou B, Hadjadj S, Wargny M, Pichelin M, Al-Salameh A, Allix I, et al. CORONADO investigators. Phenotypic characteristics and prognosis of inpatients with COVID-19 and diabetes: the CORONADO study. Diabetologia 2020;63(8):1500-1515. DOI: 10.1007/s00125020-05180-x.

4. Magoon R. Pulmonary vasculature in COVID-19: mechanism to monitoring! Korean J Anesthesiol 2021;74(2):186-187. DOI: 10.4097/ kja.20536.

5. Fabbri A, Marchesini G, Benazzi B, Morelli A, Montesi D, Bini C, et al. Stress hyperglycemia and mortality in subjects with diabetes and sepsis. Crit Care Explor 2020;2(7):e0152. DOI: 10.1097/ CCE. 0000000000000152 . 